\title{
Politische Implikationen der kulturellen Evolution
}

\section{Charakteristika und Bedeutung der kulturellen Evolution}

Bis vor rund 250 Jahren lebten 90\% der Menschheit in bitterer Armut; ihr Lebensstandard verharrte über die Jahrhunderte auf einem extrem niedrigen Niveau. Ebenso blieben die Bevölkerungszahlen über die Jahrhunderte gering; so lebten in ganz Westeuropa im Jahre 1000 lediglich rund 25 Millionen Menschen - nicht mehr als um Christi Geburt. Zu einer nachhaltigen Besserung kam es erst infolge der Mitte des 18. Jahrhunderts zunächst in Großbritannien einsetzenden Industriellen Revolution, die wenig später auch auf den westeuropäischen Kontinent und Nordamerika übergriff ${ }^{1}$. Die betreffenden Länder erlebten eine rapide, bis dahin völlig unbekannte Zunahme ihres Wohlstands und ihrer Bevölkerung. In Westeuropa beispielsweise stieg das reale Bruttoinlandsprodukt pro Kopf von 1700 bis 2001 auf das 19fache, die Bevölkerungszahl erhöhte sich bis 2001 auf 392 Millionen².

Die Industrielle Revolution und die Zunahme des Wohlstands und der Bevölkerung in der westlichen Welt seit dieser Revolution waren die Folge einer weitgehenden Durchsetzung bestimmter Institutionen: des Prinzips der individuellen Freiheit (vor allem der Vertrags-, Handels- und Gewerbefreiheit), der Dominanz und des wirksamen staatlichen Schutzes des Privateigentums (auch und gerade an Produktionsmitteln) sowie der Herrschaft des Rechts. Die Steuern waren niedrig, und der Staat verzichtete weitgehend auf eine Gängelung der Wirtschaft durch Regulierungen. Feudalismus, Zunftwesen und Merkantilismus wurden endgültig überwunden. Freies privates Unternehmertum gewann im Wirtschaftsleben die Oberhand. Die Märkte waren offen, der Wettbewerb entsprechend intensiv. Erstmals in der Geschichte wurden den Menschen umfassend Freiräume für eigenständiges, produktives Wirtschaften eröffnet, ihre wirtschaftlichen Aktivitäten zugleich effizient koordiniert.

1 Zur Industriellen Revolution siehe Thomas S. Ashton, The Industrial Revolution 17601830, London/Oxford 1969; R. M. Hartwell, The Industrial Revolution and Economic Growth, London 1971; Douglass C. North / Robert Paul Thomas, The Rise of the Western World. A New Economic History, Cambridge 1973; Nathan Rosenberg / Luther E. Birdzell, How the West Grew Rich. The Economic Transformation of the Industrial World, London 1986; Douglass C. North / Barry R. Weingast, »Constitutions and Commitment: The Evolution of Institutions Governing Public Choice in Seventeenth-Century England « in: Journal of Economic History, Vol. 49 (1989), No. 4, S. 803-832; David S. Landes, The Wealth and Poverty of Nations. Why Some Are So Rich and Some So Poor, New York/London 1998.

2 Vgl. Angus Maddison, The World Economy: Historical Statistics, Paris 2003, S. 256, 262. 
Die für die Industrielle Revolution und den dadurch ausgelösten Aufstieg der westlichen Welt entscheidenden Institutionen hatten sich zuvor im Laufe der kulturellen Evolution allmählich und ungeplant entwickelt ${ }^{3}$. Zwar wurde jede dieser Institutionen immer wieder von Menschen bewusst verändert, doch diese bewussten Änderungen mussten sich genauso wie die unbewussten im Wettbewerbsprozess

3 Die Theorie der kulturellen Evolution, die die Grundlage der vorliegenden Analyse darstellt, wurde maßgeblich von Friedrich A. von Hayek entwickelt. Siehe vor allem: Friedrich A. von Hayek, »Bemerkungen über die Entwicklung von Systemen von Verhaltensregeln« in: ders., Freiburger Studien. Gesammelte Aufsätze, Tübingen 1969, S. 144-160; ders., Recht, Gesetzgebung und Freibeit. Eine neue Darstellung der liberalen Prinzipien der Gerechtigkeit und der politischen Ökonomie (1973-79), 3 Bde., Landsberg am Lech 1981 (Bde. II \& III) und 1986 (Bd. I, 2. Aufl.); ders. »Die überschätzte Vernunft « in: Rupert J. Riedl / Franz Kreuzer (Hg.), Evolution und Menschenbild, Hamburg 1983, S. 164-192; ders., The Fatal Conceit. The Errors of Socialism, Chicago (Ill.) 1988. Wichtige Beiträge zur Theorie der kulturellen Evolution haben darüber hinaus geleistet: David Hume, »A Treatise of Human Nature: Being an Attempt to Introduce the Experimental Method of Reasoning into Moral Subjects « Vol. III: »Of Morals « (1740) in: ders., The Philosophical Works, Vol. II, Aalen 1992, S. 229-374; Adam Smith, »Considerations Concerning the First Formation of Languages, and the Different Genius of Original and Compounded Languages " (1761) in: ders., Lectures on Rhetoric and Belles Lettres, Indianapolis (Indiana) 1985, S. 201-226; ders., Der Woblstand der Nationen. Eine Untersuchung seiner Natur und seiner Ursachen (1776), München 1974; Adam Ferguson, Versuch über die Geschichte der bürgerlichen Gesellschaft (1767), Frankfurt am Main 1986; Wilhelm von Humboldt, »Ueber die Verschiedenheit des menschlichen Sprachbaues und ihren Einfluss auf die geistige Entwicklung des Menschengeschlechts « (1836) in: ders., Werke in fünf Bänden, Bd. III: Schriften zur Sprachphilosophie, 7. Aufl., Stuttgart 1994, S. 368-756; Friedrich Carl von Savigny, System des heutigen Römischen Rechts, Berlin 1840-51; Henry Sumner Maine, Das alte Recht »Ancient Law". Sein Zusammenhang mit der Frühgeschichte der Gesellschaft und sein Verhältnis zu modernen Ideen (1861), Baden-Baden 1997; ders., Lectures on the Early History of Institutions, New York 1888; Charles Darwin, Die Abstammung des Menschen (1871), 3. Aufl., Wiesbaden 1966; Carl Menger, Untersuchungen über die Methode der Socialwissenschaften, und der Politischen Oekonomie insbesondere, Leipzig 1883; L. T. Hobhouse, Morals in Evolution. A Study in Comparative Ethics (1906), London 1951; A. M. Carr-Saunders, The Population Problem. A Study in Human Evolution, Oxford 1922; Armen A. Alchian, »Uncertainty, Evolution, and Economic Theory « in: Journal of Political Economy, Vol. 58 (1950), No. 3, S. 211-221; Leslie A. White, The Evolution of Culture. The Development of Civilization to the Fall of Rome, New York 1959; Carroll Quigley, The Evolution of Civilizations. An Introduction to Historical Analysis (1961), Indianapolis (Indiana) 1979; Donald T. Campbell, »Variation and Selective Retention in Socio-Cultural Evolution « in: Herbert R. Barringer / George I. Blanksten / Raymond W. Mack (Hg.), Social Change in Developing Areas. A Reinterpretation of Evolutionary Theory, Cambridge (Mass.) 1965, S. 19-49; ders., »Evolutionary Epistemology « (1974), in: Gerard Radnitzky / W. W. Bartley (Hg.), Evolutionary Epistemology, Rationality, and the Sociology of Knowledge, La Salle (Ill.) 1987, S. 47-89; ders., »On the Conflict between Biological and Social Evolution and between Psychology and Moral Tradition « in: American Psychologist, Vol. 30 (1975), No. 12, S. 11031126; ders., »The Two Distinct Routes beyond Kin Selection to Ultrasociality: Implications for the Humanities and Social Sciences « in: Diane L. Bridgeman (Hg.), The Nature of Prosocial Development. Interdisciplinary Theories and Strategies, New York 1983, S. 11-41; Alexander Alland, Evolution and Human Behavior, Garden City (New 
der kulturellen Evolution unter ständig wandelnden, nicht prognostizierbaren Umfeldbedingungen bewähren. Welche Rechtsnormen sich beispielsweise durchsetzen würden, konnten auch diejenigen nicht voraussehen, die bestimmte Rechtsnormen bewusst erfanden und einführten. Tatsächlich wurden die wohlfahrtssteigernden Wirkungen der genannten Institutionen von denen, die sie einführten, meist nicht vorausgesehen. So gingen die individuelle Freiheit und die Herrschaft des Rechts in England im 17. Jahrhundert lediglich als Nebenprodukt aus dem seinerzeitigen Machtkampf zwischen König und Parlament hervor, der mit der Glorreichen Revolution von 1688 zugunsten des Parlaments und damit zugunsten jener Institutionen entschieden wurde ${ }^{4}$.

Die westlichen Gesellschaften, in denen sich - auf welchen Wegen auch immer produktivere Institutionen entwickelten, prosperierten und expandierten ${ }^{5}$. Sie zogen dabei Menschen anderer Gesellschaften an und verdrängten diese anderen Gesellschaften tendenziell, soweit diese nicht die produktiveren Institutionen übernahmen. Diejenigen Gesellschaften, die an weniger produktiven Institutionen festhielten oder solche einführten, fielen im Wettbewerbsprozess der kulturellen Evolution zurück. Einige liefen sogar Gefahr unterzugehen. Beispielhaft genannt seien die Indianergesellschaften Nordamerikas, die Aztekenkultur Mittelamerikas sowie das Reich der Inka in Südamerika. Ein weiteres Beispiel ist das chinesische Kaiserreich, das Westeuropa bis ins 15. Jahrhundert technologisch überlegen war. Doch die zentralistische, totalitäre und repressive Willkürherrschaft des Kaisers und

York) 1967; Karl R. Popper, Objektive Erkenntnis. Ein evolutionärer Entwurf (1972), Hamburg 1984; Clifford Geertz, The Interpretation of Cultures, New York 1973; Elman R. Service, Ursprünge des Staates und der Zivilisation. Der Prozess der kulturellen Evolution, Frankfurt am Main 1977; Wolfgang Wickler / Uta Seibt, Das Prinzip Eigennutz. Zur Evolution sozialen Verbaltens (1977), München/Zürich 1991; Edna Ullmann-Margalit, The Emergence of Norms, Oxford 1977; John C. Eccles, Das Rätsel Mensch. Die Evolution des Menschen und die Funktion des Gehirns (1979), München/ Zürich, 1989; ders., Die Evolution des Gehirns - die Erschaffung des Selbst (1989), 3. Aufl., München/Zürich 1994; H. Ronald Pulliam / Christopher Dunford, Programmed to Learn. An Essay on the Evolution of Culture, New York 1980; John Tyler Bonner, The Evolution of Culture in Animals, Princeton (New Jersey) 1980; Charles J. Lumsden / Edward O. Wilson, Genes, Mind, and Culture. The Coevolutionary Process, Cambridge (Mass.)/London 1981; Luigi Luca Cavalli-Sforza / Marcus W. Feldman, Cultural Transmission and Evolution: A Quantitative Approach, Princeton (New Jersey) 1981; Robert Axelrod, The Evolution of Cooperation, New York 1984; Robert Boyd / Peter J. Richerson, Culture and the Evolutionary Process, Chicago (Ill.) 1985; C. R. Hallpike, The Principles of Social Evolution, Oxford 1986; Allen W. Johnson / Timothy Earle, The Evolution of Human Societies. From Foraging Group to Agrarian State, Stanford (Cal.) 1987; Elinor Ostrom, Governing the Commons. The Evolution of Institutions for Collective Action, Cambridge 1990; William H. Durham, Coevolution. Genes, Culture, and Human Diversity, Stanford (Cal.) 1991; Stephen K. Sanderson, Social Transformations. A General Theory of Historical Development, Lanham (Maryland) 1999.

$4 \mathrm{Vgl}$. David Hume, The History of England from the Invasion of Julius Caesar to The Revolution in 1688 (1754-61), Indianapolis (Indiana) 1983-85; Thomas B. Macaulay, Die Glorreiche Revolution. Geschichte Englands 1688/89 (1849), Zürich 1998.

5 Vgl. Wolfgang Reinhard, Geschichte der europäischen Expansion, 4 Bde., Stuttgart 1983-90. 
seiner allmächtigen Mandarine, die aus Angst um ihre Machtstellung Privateigentum, individuelle Freiheit, privates Unternehmertum und offene Märkte verboten und sämtliche individuelle Initiative unterdrückten, führte unweigerlich zu wirtschaftlichem Rückschritt und kulturellem Verfall ${ }^{6}$. Ein aktuelles Beispiel liefern die sozialistischen Staaten des 20. Jahrhunderts. Hier ersetzten die kommunistischen Herrscher sämtliche Institutionen, die sich im Laufe der kulturellen Evolution spontan entwickelt und den Aufstieg der westlichen Welt ermöglicht hatten, durch neu konstruierte. So trat die zentrale Planung und Lenkung aller Wirtschaftsprozesse an die Stelle des freien Marktes, das staatliche an die Stelle des privaten Eigentums an den Produktionsmitteln. Jede der Institutionen, die die Kommunisten als Ersatz für die von ihnen abgeschafften einführten, wies erhebliche Funktionsmängel auf und trug damit zum schließlichen Zusammenbruch der sozialistischen Systeme bei'.

Wie der in historischer Perspektive vergleichsweise rasche Zusammenbruch der sozialistischen Systeme des 20. Jahrhunderts zeigt, sind die Gesellschaftssysteme heutzutage einem besonders starken Selektionsdruck ausgesetzt. Hierbei spielen die im Laufe des 19. und 20. Jahrhunderts deutlich gesunkenen Transaktions- und Transportkosten sowie die dadurch gestiegene Mobilität von Mensch und Kapital eine entscheidende Rolle. Praktisch überall auf der Welt können sich die Menschen heutzutage ohne große Kosten über die Zustände in anderen Ländern informieren, etwa über den dortigen Lebensstandard oder den Umfang individueller Freiheitsrechte. Auch wandern Mensch und Kapital heute schneller und in größerem Umfang als je zuvor dorthin, wo sie höhere Einkommen erzielen können. Weniger wettbewerbsfähige Gesellschaften fallen dabei unweigerlich immer weiter zurück. Die (Opportunitäts-) Kosten einer die Wettbewerbsfähigkeit der jeweiligen Gesellschaft nicht angemessen berücksichtigenden Politik sind daher heutzutage wahrscheinlich so groß wie nie zuvor in der Geschichte der Menschheit.

Welche Lehren können aus diesen Charakteristika der kulturellen Evolution für die Politik gezogen werden? An welchen Leitlinien sollte sie sich orientieren, damit die jeweilige Gesellschaft im Wettbewerbsprozess der kulturellen Evolution bestehen kann? Bevor diese Fragen beantwortet werden können, müssen zunächst zwei weitverbreitete Irrtümer über den Spielraum und die Rolle der Politik in der kulturellen Evolution ausgeräumt werden (Abschnitt 2). Anschließend werden aus den Charakteristika der kulturellen Evolution drei konkrete Leitlinien abgeleitet, die die Politik beachten sollte (Abschnitt 3).

6 Vgl. David S. Landes, aaO. (FN 1), S. 55 ff.; Erich Weede, Asien und der Westen. Politische und kulturelle Determinanten der wirtschaftlichen Entwicklung, Baden-Baden 2000, S. $86 \mathrm{ff}$.

7 Vgl. Horst Feldmann, »Kulturelle Evolution und der Zusammenbruch des Sozialismus« in: List Forum für Wirtschafts- und Finanzpolitik, Bd. 23 (1997), Nr. 1, S. 82-101. 


\section{Zwischen Panglossismus und Konstruktivismus}

\subsection{Die Gefahr des Panglossismus}

In Voltaires Roman »Candide« behauptet der ehrwürdige Hofmeister und Hauslehrer Dr. Pangloß, wir lebten in der besten aller möglichen Welten: »»Es ist erwiesen«, pflegte er zu sagen, »daß die Dinge gar nicht anders sein können, als sie sind. Denn sintemal alles zu einem ganz bestimmten $Z$ weck geschaffen ist, so ist alles notwendigerweise zum allerbesten Zweck erschaffen ««8. Die Auffassung, wir lebten in der besten aller möglichen Welten, wenn man nur alle Probleme und Widerstände berücksichtige, die denkbaren Verbesserungen entgegenstünden, wird dementsprechend heute als Panglossismus bezeichnet. Kritiker einer kulturevolutionären Sicht der Welt behaupten, diese Sicht impliziere einen solchen kritiklosen Panglossismus. Aus kulturevolutionärer Sicht sei alles, was im Laufe der kulturellen Evolution überlebt habe, überlegen; indem es überlebt habe, habe es sich als überlegen erwiesen. Der Theorie der kulturellen Evolution fehle damit ein unabhängiger Maßstab zur Bewertung der Ergebnisse der kulturellen Evolution und zur Bewertung politischer Reformvorschläge. Auch ließen sich aus ihr keinerlei politische Reformvorschläge ableiten. Die Vertreter der Theorie schlössen implizit vom "Sein« auf das "Sollen« und seien damit einem naturalistischen Trugschluss erlegen. Konsequenterweise müssten selbst totalitäre Regime, die im Laufe der kulturellen Evolution entstanden seien, akzeptiert werden. Die Theorie der kulturellen Evolution sei fatalistisch'

Tatsächlich besteht die Gefahr des Panglossismus jedoch nicht. Die Vertreter der Theorie der kulturellen Evolution sind keinem naturalistischen Fehlschluss erlegen. Sie behaupten nicht, die Ergebnisse der kulturellen Evolution seien notwendigerweise »gut «, sondern vielmehr, dass die Menschheit in ihrer heutigen Größe und mit ihrem heutigen Wohlstand ohne die erwähnten spezifischen Institutionen und deren weitere Evolution nicht fortbestehen könnte. Würden diese Institutionen zerstört,

8 Voltaire, Candide oder Der Glaube an die beste der Welten (1759), München 1980, S. 8.

9 Vgl. James M. Buchanan, »Möglichkeiten institutioneller Reformen im Rahmen kulturell geformter abstrakter Verhaltensregeln « in: Viktor Vanberg, Liberaler Evolutionismus oder vertragstheoretischer Konstitutionalismus? Zum Problem institutioneller Reformen bei F. A. von Hayek und J. M. Buchanan, Tübingen 1981, S. 45 f.; ders., Die Grenzen der Freibeit. Zwischen Anarchie und Leviathan, Tübingen 1984, S. 237; Geoffrey Brennan / James M. Buchanan, The Reason of Rules. Constitutional Political Economy, Cambridge 1985, S. 9 f.; David Miller, »The Fatalistic Conceit « in: Critical Review, Vol. 3 (1989), No. 2, S. 314; Stefan Voigt, »On the Internal Consistency of Hayek's Evolutionary Oriented Constitutional Economics - Some General Remarks « in: Journal des Economistes et des Etudes Humaines, Vol. 3 (1992), No. 4, S. 465; Martin De Vlieghere, "A Reappraisal of Friedrich A. Hayek's Cultural Evolutionism « in: Economics and Philosophy, Vol. 10 (1994), No. 2, S. 293; John N. Gray, Freibeit im Denken Hayeks, Tübingen 1995, S. 141. Eine kritische Auseinandersetzung mit dieser und weiterer Kritik, die an der Theorie der kulturellen Evolution geübt wird, findet sich bei Horst Feldmann, »Hayeks Theorie der kulturellen Evolution: Eine Kritik der Kritik « in: Thomas Eger (Hg.), Kulturelle Prägungen wirtschaftlicher Institutionen und wirtschaftspolitischer Reformen, Berlin 2002, S. 51-88. 
würde dies einen Großteil der Menschheit zu Armut und Hungertod verurteilen. Die Vorzüge der genannten Institutionen rechtfertigen diese zwar nicht per se, aber die Alternative bestünde für viele Menschen in Armut und Tod ${ }^{10}$.

Akzeptiert man die Erhaltung der Menschheit und ihres Wohlstands als normativen Standard, lassen sich aus der Theorie der kulturellen Evolution sehr wohl konkrete politische Gestaltungsempfehlungen ableiten. Politische Reformen sind nicht nur möglich, sondern auch nötig, denn obgleich die Institutionen im Prozess der kulturellen Evolution grundsätzlich nach ihrem Beitrag zum Überleben und zum Wohlstand der Menschen selektiert werden, wird diese Entwicklung zugleich immer wieder durch diverse Faktoren gestört. So bestehen beispielsweise viele überlieferte Institutionen noch fort, wenn sie ihre Nützlichkeit schon lange verloren haben, und sogar, wenn sie mehr ein Hindernis als eine Hilfe geworden sind - etwa dann, wenn sie nicht mit neuen Problemen fertig werden können. Auch entwickeln sich einzelne Institutionen, wie etwa die richterliche Rechtsfortbildung, bisweilen in kontraproduktive Richtungen, was eine Korrektur durch bewusste Gesetzgebung erforderlich macht. Wie die Geschichte zeigt, kann der kulturelle Evolutionsprozess sogar ganze Gesellschaften in Sackgassen führen, aus denen sie sich nur schwer wieder befreien können.

Des weiteren ist es bisweilen notwendig, gewachsene Institutionen gezielt zu verbessern, um ihr volles Potential zu erschließen. So ist beispielsweise die Institution des Privateigentums, wie sie heute existiert, bei weitem noch nicht perfekt. Beim Eigentum an einem Gut handelt es sich um ein komplexes Bündel an Rechten, die auf unterschiedliche Weise ausgestaltet und verschiedenen Personen oder Personengemeinschaften zugeordnet werden können ${ }^{11}$. Die verschiedenen Möglichkeiten der Ausgestaltung und Zuordnung von Eigentumsrechten sind noch lange nicht ausgeschöpft (etwa im Bereich von Finanzierungsinstrumenten oder naturwissenschaftlichen Entdeckungen und Erfindungen). Der Gesetzgeber muss die Voraussetzungen dafür schaffen, dass diese Möglichkeiten voll genutzt werden können.

Gestört wird die kulturelle Evolution darüber hinaus immer wieder durch einen mächtigen Staat. Mächtige Regierungen tendieren immer wieder dazu, ihre Macht zu missbrauchen, indem sie ihre Bürger unterdrücken und den Prozess der kulturellen Evolution aufzuhalten oder zu lenken versuchen. Man denke an die Beispiele des chinesischen Kaiserreichs und der sozialistischen Regime des 20. Jahrhunderts. Auch die heutigen repräsentativen Demokratien gefährden die überlieferten Institutionen des Rechtsstaats und der Marktwirtschaft. Da die Macht der Politiker in den heutigen Demokratien unzulänglich beschränkt ist und sie auf die Unterstützung durch organisierte Interessengruppen angewiesen sind, wird immer wieder unter Verletzung rechtsstaatlicher und marktwirtschaftlicher Prinzipien zugunsten solcher Interessengruppen interveniert, wodurch die freiheitliche Ordnung des Wes-

10 Vgl. Friedrich A. von Hayek, The Fatal Conceit. The Errors of Socialism, aaO. (FN 3), S. $27,63$.

11 Vgl. Horst Feldmann, Ordnungstheoretische Aspekte der Institutionenökonomik, Berlin 1999, S. $54 \mathrm{ff}$. 
tens gefährdet wird ${ }^{12}$. Auch die Idee der »sozialen Gerechtigkeit « gefährdet heutzutage die Funktionsfähigkeit dieser Ordnung, weil sie immer wieder zum Anlass für ordnungsinkonforme Interventionen genommen wird ${ }^{13}$. Daher sind ordnungspolitische Maßnahmen notwendig, die den Machtmissbrauch der Regierungen und den schädlichen Einfluss der Interessengruppen möglichst effektiv unterbinden.

Ein weiterer Faktor, der die kulturelle Evolution immer wieder stört und dem daher durch geeignete politische Maßnahmen begegnet werden muss, sind die angeborenen Instinkte der Menschen. Über Millionen von Jahren haben der Mensch und seine hominiden Vorfahren in kleinen Horden zusammengelebt, deren Mitglieder sich persönlich kannten. Das Zusammenleben in einer solchen Gruppe, die von einem Anführer geleitet wurde, war durch gemeinsame, konkrete Ziele und eine gleichartige Wahrnehmung der Geschehnisse gekennzeichnet, die den Gruppenmitgliedern gemeinsam sichtbar und von allen als potentielle Quelle von Nahrung oder Gefahr erkannt wurden. Die Kooperation innerhalb der Gruppe war eng umschrieben. In dieser Zeit entwickelten und verfestigten sich bestimmte genetisch vererbte Instinkte, die das Verhalten der Menschen leiteten. Sie waren dem Leben in der Kleingruppe angepasst und auf die Sicherung ihres Zusammenhalts und Fortbestands ausgerichtet. Wichtige Beispiele sind die Instinkte der gleichmäßigen Einkommensverteilung, der Solidarität und des Altruismus, die nicht auf alle Menschen gerichtet waren, sondern nur auf die Mitglieder der eigenen Gruppe. Im Zuge der kulturellen Evolution wurden diese angeborenen Instinkte immer mehr durch erlernte Verhaltensregeln beherrscht und verdrängt ${ }^{14}$. Nur dadurch ist die Entstehung einer ausgedehnten Gesellschaftsordnung möglich geworden. Von Zeit zu Zeit lehnen sich die unterdrückten Instinkte indes gegen die Disziplin der anerzogenen Verhaltensnormen auf, etwa gegen die Regeln der Vertragstreue oder des Respekts vor der Freiheit und dem Eigentum anderer. Beispielhaft kommt dies in den Lehren der Kommunisten zum Ausdruck. Sie verdammten diese Normen und appellierten statt dessen an die Urinstinkte der Gleichverteilung, der Solidarität und des Altruismus. Bei einer solchen Auflehnung besteht die Gefahr, dass die Menschen wieder in die Vorstellungen der Stammesgesellschaft zurückfallen und damit den Bestand der Gesellschaftsordnung bedrohen, der sie nicht nur ihren Wohlstand, sondern sogar ihr Leben verdanken ${ }^{15}$.

12 Vgl. Mancur Olson, The Logic of Collective Action. Public Goods and the Theory of Groups, Cambridge (Mass.) 1965; ders., The Rise and Decline of Nations. Economic Growth, Stagflation and Social Rigidities, New Haven (Conn.), London 1982; Fareed Zakaria, The Future of Freedom. Illiberal Democracy at Home and Abroad, New York 2003.

13 Vgl. Friedrich A. von Hayek, Recht, Gesetzgebung und Freibeit. Eine neue Darstellung der liberalen Prinzipien der Gerechtigkeit und der politischen Ökonomie, Bd. II: Die Illusion der sozialen Gerechtigkeit, aaO. (FN 3).

$14 \mathrm{Vgl}$. Friedrich A. von Hayek, »Die überschätzte Vernunft« aaO. (FN 3); ders., The Fatal Conceit. The Errors of Socialism, aaO. (FN 3). 


\subsection{Die Gefahr des Konstruktivismus}

Anders als der Panglossismus, der nur eine scheinbare Gefahr darstellt, stellt der Konstruktivismus (oder auch konstruktivistische Rationalismus) eine reale Gefahr dar. Die Vertreter dieser Richtung behaupten, gesellschaftliche Institutionen könnten menschlichen Zwecken nur dienen, wenn sie absichtlich für diese Zwecke geschaffen worden seien. Institutionen, die nicht rational entworfen worden seien, könnten nur durch Zufall wohltätig sein ${ }^{16}$. Irgendwann im Laufe der Evolution sei die Vernunft entstanden, und seitdem habe der Mensch die gesellschaftlichen Institutionen immer systematischer rational gestaltet. Die Vertreter des Konstruktivismus sind der Überzeugung, sämtliche Institutionen - einschließlich der Sprache, der Moral und des Rechts - und damit die gesamte Gesellschaftsordnung könnten mit Hilfe der Vernunft neu konstruiert werden. Die Gesellschaft und ihre Institutionen sollten so umgestaltet werden, dass sie bestimmten festgelegten Zwecken bestmöglich dienten.

Seit der Aufklärung ist dieser konstruktivistische Glaube die dominierende Geisteshaltung der meisten Menschen, insbesondere der Intellektuellen und der Politiker. In besonders reiner Form zeigt er sich in den Lehren der Kommunisten. Diese versuchten im 20. Jahrhundert in vielen Ländern, eine vollkommen neue Gesellschaftsordnung mit rational entworfenen Institutionen aufzubauen und dabei alle traditionellen Institutionen, die ihrer Ansicht nach ohnehin nur der Ausbeutung der Arbeiterklasse dienten, abzuschaffen. So wurde nicht nur der freie Markt durch die Zentralplanung ersetzt und das Privateigentum an den Produktionsmitteln durch das Staatseigentum; darüber hinaus wurde die Herrschaft des Rechts durch ein sozialistisches Recht ersetzt, das nicht der Gewährleistung individueller Freiheitsrechte, sondern der Umsetzung der Direktiven der regierenden kommunistischen Partei diente. Selbst die traditionellen Moralvorstellungen der westlichen Zivilisation sollten durch eine neue, sozialistische Moral ersetzt werden. Der Sozialismus des 20. Jahrhunderts stellte den gigantischen Versuch dar, die gesamte Gesellschaft bis in ihre letzten Verästelungen nach einer umfassenden Theorie neu zu gestalten. Diesem Zweck dienten die Abschaffung sämtlicher bedeutender, spontan gewachsener Institutionen und ihr Ersatz durch neue, zielgerichtet konstruierte. Genau in dieser Hybris liegt auch der Grund für den schließlichen Zusammenbruch der sozialistischen Systeme ${ }^{17}$.

Obgleich der Konstruktivismus in der reinen Form, in der er im »real existierenden«Sozialismus verwirklicht war, kläglich gescheitert ist, ist er nach wie vor leben-

15 Vgl. Friedrich A. von Hayek, Recht, Gesetzgebung und Freibeit. Eine neue Darstellung der liberalen Prinzipien der Gerechtigkeit und der politischen Ökonomie, Bd. II: Die Illusion der sozialen Gerechtigkeit, Bd. III: Die Verfassung einer Gesellschaft freier Menschen, aaO. (FN 3).

16 Vgl. René Descartes, Discours de la Méthode / Von der Methode des richtigen Verstandesgebrauchs und der wissenschaftlichen Forschung (1637), Hamburg 1960.

17 Vgl. Horst Feldmann, »Kulturelle Evolution und der Zusammenbruch des Sozialismus«aaO. (FN 7). 
dig. Immer wieder lehnt sich die menschliche Vernunft gegen überlieferte Verhaltensregeln auf, deren Sinn sie nicht versteht. Ein Beispiel ist der im 19. Jahrhundert entstandene und auch heute noch einflussreiche Rechtspositivismus. Er hat keinerlei Verständnis für überlieferte Prinzipien und Regeln des Rechts, die nicht rational erklärt werden können und die die gesetzgeberische Macht beschränken. Für ihn besteht das Recht definitionsgemäß ausschließlich aus bewussten Befehlen des menschlichen Willens. Es soll gezielt zur Erreichung bestimmter Zwecke gestaltet werden ${ }^{18}$. Nach dem Rechtspositivismus darf die Macht des Gesetzgebers keiner Beschränkung unterworfen werden, konsequenterweise auch keiner Beschränkung durch die Respektierung individueller Freiheitsrechte. Der Rechtspositivismus wurde damit nicht nur zu einem Wegbereiter des Sozialismus und Nationalsozialismus; er hat auch das Rechtsverständnis in der westlichen Welt nachhaltig verändert.

Der konstruktivistische Glaube wird schließlich auch dadurch befördert, dass heutzutage immer mehr Menschen in großen Organisationen, wie etwa Großunternehmen oder staatlichen Behörden arbeiten. Der Erfolg der bewussten Schaffung neuer Regeln für solche Zweckorganisationen war so eindrucksvoll, dass die Bedeutung gewachsener Regeln und spontaner Ordnungen immer weniger erkannt wird. Der Verständnishorizont der Menschen, die in solchen großen, für einen bestimm-

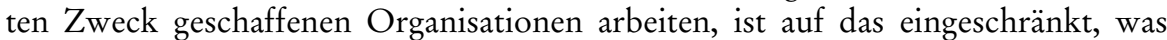
durch die interne Struktur derartiger Organisationen erfordert wird. Für sie ist die spontane Handelnsordnung einer ausgedehnten, marktwirtschaftlichen Gesellschaft weitgehend unverständlich; sie haben die Regeln, auf denen sie beruht, niemals angewandt; ihre Funktionsweise und ihre Verteilungswirkungen erscheinen ihnen irrational und unmoralisch. Daher fordern sie, dass die gesamte Gesellschaftsordnung und deren Institutionen auf dieselbe Weise konstruiert werden sollten wie eine Großorganisation und dass damit insbesondere eine gerechte Einkommensverteilung erreicht werden sollte.

Anders als der Konstruktivismus behauptet, können eine Gesellschaftsordnung und ihre Institutionen jedoch nicht gänzlich neu konstruiert werden. Die kulturelle Evolution insgesamt lässt sich nicht vernunftmäßig steuern. Die Vernunft selbst ist erst im Laufe der kulturellen Evolution entstanden. Sie hat den Prozess der kulturellen Evolution nicht gelenkt, sondern hat sich selbst erst in der Frühphase dieses Prozesses allmählich entwickelt ${ }^{19}$. Der Mensch hat neue Verhaltensregeln nicht angenommen, weil er intelligent war; vielmehr wurde er dadurch intelligent, dass er sich neuen Verhaltensregeln unterwarf. Der Mensch hat seine wohltätigsten Institutionen, von der Sprache bis zur Moral, dem Recht und dem Markt, nicht erfunden; dazu war er nicht intelligent genug.

Auch heute noch kann der Mensch die Bedeutung und Interdependenz der Institutionen, die sein Verhalten leiten, nicht vollständig erfassen. Er weiß meist nicht, aus welchen Gründen sie ursprünglich entstanden sind, warum sie sich im Vergleich $\mathrm{zu}$ anderen besser bewährt haben, welchen Beitrag sie zur Erhaltung der komplexen Handelnsordnung einer großen Gesellschaft leisten, warum und auf welche Weise 
sie sich im Zeitablauf wandeln und inwiefern sie voneinander abhängen. Vielfach sind die Verhaltensregeln den Menschen noch nicht einmal bewusst, obwohl sie sich nach ihnen richten. Auch im Rahmen eingehender wissenschaftlicher Analysen können die Entstehung, die Funktionen, der Wandel und die wechselseitige Abhängigkeit der Institutionen einer großen Gesellschaft nur unvollständig eruiert werden. Auch lassen sich die künftigen Umfeldbedingungen, an die sich die Institutionen anpassen müssen, nicht vorhersehen. Aus all diesen Gründen kann die kulturelle Evolution nicht gelenkt oder kontrolliert werden. Der Irrtum der Konstruktivisten besteht in dem Glauben, all dieses Wissen in Erfahrung bringen und auf seiner Grundlage eine vollkommen neue Gesellschaftsordnung errichten zu können. Wie das Beispiel der sozialistischen Regime des 20. Jahrhunderts zeigt, würde ein solcher Versuch aber den inzwischen erreichten Wohlstand der Menschheit und sogar die physische Existenz eines Teils der heutigen Weltbevölkerung gefährden, weil dabei zwangsläufig traditionelle Institutionen zerstört würden, die für die Erhaltung der heutigen Weltbevölkerung und ihres Wohlstands unerlässlich sind.

Dies alles bedeutet selbstverständlich nicht, dass die überlieferten Institutionen nicht verbessert werden können. Da man sie nicht in vollem Umfang verstehen kann, sollte man sie nur nicht unbesehen abschaffen, sondern im Falle erkennbarer Mängel prüfen, inwiefern sich durch die Änderung einer Institution die Funktionsweise der Gesamtheit aller Institutionen voraussichtlich verbessern und die Erhaltung der komplexen Ordnung menschlichen Handelns in einer großen Gesellschaft besser unterstützen lässt. Auf der Grundlage solcher Analysen können dann politische Reformen durchgeführt werden.

19 "Ein Repertoire erlernter Regeln, die ihm sagten, was richtiges und was falsches Handeln unter verschiedenen Umständen sei, gab ihm in zunehmendem Maße die Fähigkeit, sich an wechselnde Bedingungen anzupassen - und insbesondere mit den anderen Mitgliedern seiner Gruppe zu kooperieren. So begann eine Tradition von Verhaltensregeln das menschliche Dasein zu regulieren, die unabhängig von jedem einzelnen Individuum galten, das sie erlernt hatte. Als diese durch Lernen erworbenen Regeln, die auch Klassifizierungen der verschiedenen Arten von Objekten einschlossen, anfingen, mit der Zeit eine Art Umweltmodell miteinzubegreifen, das den Menschen befähigte, äußere Ereignisse vorauszusagen und bei seinem Handeln zu antizipieren, erschien das, was wir als Vernunft bezeichnen. Dieses System von Verhaltensregeln enthielt damals wahrscheinlich viel mehr »Intelligenz « als das Denken des Menschen über seine Umwelt. [. . .] Der Geist ist in eine traditionelle unpersönliche Struktur erlernter Regeln eingebettet, und seine Fähigkeit, Erfahrungen zu ordnen, ist eine erworbene Wiederholung kultureller Muster, die jeder individuelle Geist als Gegebenheit vorfindet. Das Gehirn ist ein Organ, das uns befähigt, Kultur aufzunehmen, aber nicht, sie zu entwerfen. «Friedrich A. von Hayek, Recht, Gesetzgebung und Freibeit. Eine neue Darstellung der liberalen Prinzipien der Gerechtigkeit und der politischen Ökonomie, Bd. III: Die Verfassung einer Gesellschaft freier Menschen, aaO. (FN 3), S. 213 f. 


\section{Leitlinien für die Politik}

Wie die bisherigen Ausführungen bereits deutlich machen, spielt die Politik im Rahmen der kulturellen Evolution eine bedeutende Rolle. Angesichts des Selektionsdrucks, der gerade heute in der kulturellen Evolution herrscht, sollte sie sich dabei für Maßnahmen entscheiden, die für die Erhaltung und den Wohlstand der jeweiligen Gesellschaft, für die die Politik Verantwortung trägt, förderlich sind. Die Entscheidung für ein solches Ziel impliziert zwar ein Werturteil, das nicht wissenschaftlich bewiesen werden kann, jedoch muss sich jede Politik an bestimmten, wissenschaftlich nicht beweisbaren Zielen orientieren ${ }^{20}$. Für das genannte Ziel spricht, dass seine Erreichung die Voraussetzung darstellt für die Erreichung praktisch aller anderen Ziele, die die Mitglieder der jeweiligen Gesellschaft haben mögen. Außerdem dürfte es von praktisch allen Menschen bejaht werden.

Aus den Charakteristika der kulturellen Evolution können, wenn man das genannte Ziel akzeptiert, allgemeine Leitlinien für die Politik deduziert werden. Die Beachtung dieser im folgenden entwickelten Leitlinien erleichtert die Erreichung jenes Ziels. Zudem gewährleisten sie Einheitlichkeit und innere Widerspruchslosigkeit der Politik. Dadurch werden kostenträchtige Friktionen vermieden, die ohne Beachtung allgemeiner Leitlinien aufträten.

Die im folgenden entwickelten Leitlinien haben einen abstrakten Charakter, denn sie legen nur einige Aspekte der Politik fest. Damit belassen sie für die konkrete Gestaltung politischer Institutionen und praktischer politischer Maßnahmen einen großen Spielraum, der gemäß den von Land zu Land unterschiedlichen geschichtlichen Erfahrungen, kulturellen Werten und Traditionen, Umfeldbedingungen und Präferenzen der Menschen auf verschiedene Weise ausgefüllt werden kann. Die Leitlinien selbst hingegen sind nicht beliebig. Sie ergeben sich logisch schlüssig aus den Charakteristika der kulturellen Evolution. Sollen die Existenz der jeweiligen Gesellschaft und ihr Wohlstand gesichert werden, müssen sie eingehalten und in konkrete politische Maßnahmen und Institutionen umgesetzt werden. Je konsequenter dies geschieht, desto größer der Zielerreichungsgrad. Je weniger es geschieht, desto größer die Gefährdung nicht nur des Wohlstands, sondern selbst des langfristigen Bestands der jeweiligen Gesellschaft.

\subsection{Individuelle Freibeit}

Die wichtigste Leitlinie der Politik muss angesichts der Charakteristika der kulturellen Evolution in einer umfassenden Verwirklichung individueller Freiheit bestehen. Der einzelne Mensch muss sein Leben im Rahmen seiner Möglichkeiten weitestgehend nach eigenen Vorstellungen gestalten können. Dabei hat er

20 Vgl. Max Weber, »Die »Objektivität« sozialwissenschaftlicher und sozialpolitischer Erkenntnis« (1904) in: ders., Gesammelte Aufsätze zur Wissenschaftslebre, 7. Aufl., Tübingen 1988, S. 146-214. 
selbstverständlich zugleich die Konsequenzen seiner Handlungen zu tragen (Prinzip der Haftung), denn ohne persönliche Verantwortung ist eine freiheitliche Gesellschaftsordnung nicht funktionsfähig ${ }^{21}$. Außerdem darf der einzelne bei der Verfolgung seiner Ziele nicht die Freiheit anderer Menschen verletzen ${ }^{22}$.

Individuelle Freiheit ist ein gesellschaftlicher Zustand, in dem Zwang auf Menschen von Seiten anderer so weit herabgemildert wird, als dies im Gesellschaftsleben möglich ist ${ }^{23}$. Andere dürfen nicht die Macht besitzen, den Individuen ihren Willen aufzuzwingen. Daher muss dem einzelnen ein privater Bereich gesichert sein, in den andere nicht eingreifen können. Vor allem muss der einzelne frei über eigene Mittel verfügen können; erst dadurch wird es ihm möglich, eigene Ziele zu verfolgen. Daher bildet Privateigentum ein zentrales Element der individuellen Freiheit. Es ist eine unabdingbare Voraussetzung für deren Ausübung.

Zur individuellen Freiheit gehört des weiteren die Vertragsfreiheit. Nur wenn die Gesellschaftsmitglieder grundsätzlich frei Verträge mit anderen schließen dürfen, können sie ihre Freiheit tatsächlich im Gesellschaftsleben praktizieren. Beispielsweise muss der einzelne grundsätzlich die Freiheit haben, Kaufverträge über den Erwerb derjenigen Güter abzuschließen, die er zu besitzen wünscht.

Ausdruck individueller Freiheit ist darüber hinaus die Wettbewerbsfreiheit ${ }^{24}$. Jeder einzelne muss grundsätzlich das Recht haben, seine Güter und Produktionsfaktoren auf einem Markt ungehindert anzubieten und damit in Konkurrenz zu anderen Anbietern zu treten. Die Märkte müssen offen sein. Dieses Prinzip hat nicht nur für den Bereich der Wirtschaft zu gelten, sondern auch für die Politik, die Wissenschaft, die Religion und andere wichtige Bereiche des Gesellschaftslebens. Es muss also nicht nur Berufs- und Gewerbefreiheit herrschen, vielmehr müssen auch die politische Wahlen frei und die Freiheit von Forschung und Lehre gewährleistet sein, ebenso die Glaubens-, Meinungs- und Pressefreiheit. Auch in diesen Bereichen der Gesellschaft muss damit freier Wettbewerb herrschen.

Die zentrale Bedeutung der individuellen Freiheit im Prozess der kulturellen Evolution besteht in ihren wohlfahrtssteigernden Wirkungen. Jeder einzelne hat die Möglichkeit und den Anreiz, seine Mittel so einzusetzen, dass sie einen möglichst großen Ertrag abwerfen. Wie bereits Adam Smith ${ }^{25}$ betont hat, hat ein jeder bei seinen Aktivitäten zwar nur seinen eigenen Vorteil im Auge und nicht etwa den der Gesellschaft, doch wird er durch den Wettbewerb wie von einer unsichtbaren Hand dazu geführt, dabei gleichzeitig die gesellschaftliche Wohlfahrt zu erhöhen. Der einzelne Unternehmer etwa wird dazu angehalten, Güter zu produzieren, die den Konsumentenpräferenzen entsprechen, und dabei Produktionsverfahren anzuwenden, die die größtmögliche Effizienz des Faktoreinsatzes gewährleisten. Auch hat er

21 Vgl. Walter Eucken, Grundsätze der Wirtschaftspolitik (1952), Tübingen 1990, S. 279 ff.

22 Vgl. John Stuart Mill, Über die Freiheit (1859), Stuttgart 1988, S. 16 ff., 77 f.

23 Vgl. Friedrich A. von Hayek, Die Verfassung der Freibeit (1960), 3. Aufl., Tübingen 1991, S. 13.

24 Vgl. Erich Hoppmann, Wirtschaftsordnung und Wettbewerb, Baden-Baden 1988.

25 Vgl. Adam Smith, Der Woblstand der Nationen. Eine Untersuchung seiner Natur und seiner Ursachen, aaO. (FN 3), S. $369 \mathrm{ff}$. 
einen Anreiz, auf Änderungen von Beschaffungspreisen und anderen Daten durch flexible Anpassung der Produktion zu reagieren sowie selbständig kostengünstigere Produktionsmethoden und neue, bessere Produkte zu entwickeln. Der einzelne Arbeitnehmer hat ebenfalls einen Anreiz, möglichst produktiv zu arbeiten, weil er auf diese Weise sein persönliches Einkommen steigern kann. Eine höhere Produktivität führt wiederum zu einem größeren Wohlstand der Gesellschaft.

Die wohlfahrtssteigernden Wirkungen der individuellen Freiheit sind auch darauf zurückzuführen, dass jedes Gesellschaftsmitglied einen Anreiz hat, seine persönlichen Kenntnisse und Fertigkeiten einzusetzen. Auf diese Weise wird das Wissen aller Menschen zum Wohle der ganzen Gesellschaft genutzt. Dies gilt für die Kenntnisse bestimmter Sachgebiete, Märkte, Regionen etc. ebenso wie für die unterschiedlichen Begabungen der Menschen. Die individuelle Freiheit ist ein Anreiz und ein Mechanismus zur gesellschaftlichen Nutzung solchen von Mensch zu Mensch unterschiedlichen Wissens ${ }^{26}$.

Voraussetzung für den wirtschaftliche Fortschritt sind wissenschaftliche Entdeckungen, technische Erfindungen und ökonomische Innovationen. Dabei kommt es jedoch oftmals zu Fehlschlägen. Daher ist es erforderlich, dass sich eine Vielzahl eigenständiger, schöpferischer Individuen unabhängig voneinander um Entdeckungen, Erfindungen und Innovationen bemüht. Es bedarf vieler unabhängiger Experimente in Wissenschaft, Technik und Wirtschaft, damit die Wohlfahrt der Menschen permanent erhöht werden kann. Voraussetzung dafür wiederum ist die Freiheit des Individuums. Individuen, die nach neuen Wegen suchen und mit neuen Möglichkeiten experimentieren, benötigen Freiheit, weil sie das Althergebrachte in Frage stellen und überwinden möchten. Und je mehr Individuen sich an solchen dezentralen Suchprozessen beteiligen, desto größer sind die Chancen, dass sich die gesellschaftliche Wohlfahrt erhöht ${ }^{27}$.

Die Vorteile der individuellen Freiheit gelten nicht nur für den Bereich der Wirtschaft. Sie kommen auch in der Politik, der Wissenschaft und in anderen wichtigen Bereichen des gesellschaftlichen Zusammenlebens zum Tragen. So führt der Wettbewerb alternativer Meinungen und Konzepte in der Politik zur Entwicklung überlegener Lösungen politischer Probleme. In der Wissenschaft führt die Unabhängigkeit der Forschung und der Wettbewerb zwischen den Forschern zu einem permanenten Strom neuer Erkenntnisse und zur raschen Verbreitung der bedeutendsten dieser neuen Erkenntnisse. Das Prinzip der Haftung hält Politiker und Wissenschaftler grundsätzlich genauso zu einem verantwortungsvollen Umgang mit der ihnen eingeräumten Freiheit an wie Unternehmer und Arbeitnehmer im Bereich der Wirtschaft.

26 Vgl. Friedrich A. von Hayek, »Die Verwertung des Wissens in der Gesellschaft « (1945) in: ders., Individualismus und wirtschaftliche Ordnung, 2. Aufl., Salzburg 1976, S. 103 121.

27 Vgl. Wilhelm von Humboldt, »Ideen zu einem Versuch, die Gränzen der Wirksamkeit des Staats zu bestimmen " (1851) in: ders., Werke in fünf Bänden, Bd. I: Schriften zur Anthropologie und Geschichte, 3. Aufl., Stuttgart 1980, S. 64 f.; John Stuart Mill, aaO. (FN 22), S. 97. 
Da kulturelle Evolution selbst in einem permanenten Wettbewerb unterschiedlicher Werte, Institutionen und Gruppen besteht, ist individuelle Freiheit vor allem deshalb von entscheidendem evolutionären Vorteil, weil sie Starrheit gesellschaftlicher Strukturen vermeidet und statt dessen Anpassungsfähigkeit fördert. Sämtliche Gesellschaftsmitglieder werden angehalten, sich rasch an Datenänderungen anzupassen und kontinuierlich nach neuen Problemlösungen zu suchen. Da dabei praktisch ihre gesamten Kenntnisse und Fertigkeiten genutzt werden und ein großes $\mathrm{Maß}$ an Wohlstand erwirtschaftet wird, hat eine Gesellschaft, in der das Prinzip der individuellen Freiheit umfassend verwirklicht ist, besonders gute Aussichten, im Prozess der kulturellen Evolution zu bestehen und zu prosperieren. Ihre Gesellschaftsmitglieder treiben den Prozess der kulturellen Evolution sogar aktiv mit voran - wenn auch meist unbewusst -, indem sie neue Institutionen (etwa neue Rechtsnormen oder neue Formen der Unternehmensorganisation) entwickeln und wettbewerblich testen.

Um die Vorteile der individuellen Freiheit im Prozess der kulturellen Evolution möglichst gut zu nutzen, sollte sie umfassend verwirklicht werden. Sie sollte in möglichst vielen Gesellschaftsbereichen angewandt werden (vor allem in Wirtschaft, Politik und Wissenschaft) und so ausgestaltet sein, dass sämtlichen Gesellschaftsmitgliedern ein möglichst großer Freiheitsspielraum belassen wird. Eine solche umfassende Verwirklichung individueller Freiheit bedeutet nicht, dass die kulturellen Werte und Traditionen des jeweiligen Landes und die Präferenzen seiner Bürger unberücksichtigt bleiben müssen. Im Gegenteil: Erstens können je nach den kulturellen Werten und Traditionen und der jeweiligen historischen Situation, in der sich das betreffende Land befindet, verschiedenartige Maßnahmen zur Umsetzung des Prinzips der individuellen Freiheit ergriffen werden. Zweitens und vor allem aber belässt gerade die individuelle Freiheit den Menschen einen größtmöglichen Spielraum, ihre persönlichen Ziele zu verfolgen - Ziele, die wiederum von den kulturellen Werten und Traditionen sowie von der Geschichte des jeweiligen Landes geprägt sind. Individuelle Freiheit gewährleistet einen hohen Zielerreichungsgrad; worin die konkreten Ziele der Menschen aber bestehen (welche Güter sie beispielsweise zu konsumieren wünschen), können sie selbst bestimmen.

\subsection{Herrschaft des Rechts}

Die zweite Leitlinie besagt, dass nicht Menschen, sondern das Recht herrschen sollte. Die rechtlichen Regeln müssen dabei folgenden Anforderungen genügen ${ }^{28}$ :

- Allgemeinheit: Es muss sich um langfristige Regeln handeln, die sich auf eine Vielzahl unbekannter Fälle und Personen beziehen.

- Abstraktheit: Die Regeln dürfen nur festlegen, dass die Handlungen der Individuen unter bestimmten Umständen bestimmte Bedingungen zu erfüllen haben;

28 Vgl. Friedrich A. von Hayek, Die Verfassung der Freibeit, aaO. (FN 23), S. 178 ff., 268 ff. 
alle Arten des Handelns, die diese Bedingungen erfüllen, müssen statthaft sein. Die Regeln beinhalten keine konkreten Anordnungen und gelten unabhängig von den jeweiligen Zielen der Menschen.

- Gewißheit: Die Regeln müssen bekannt und gewiß sein, um Rechtssicherheit zu gewährleisten. Sie dürfen daher auch nie rückwirkend gelten.

- Gleichheit: Die Regeln dürfen nicht gezielt bestimmte Personen begünstigen oder benachteiligen. Alle Personen müssen gleich behandelt werden. Die Regeln müssen für die Regierung ebenso gelten wie für die Regierten.

Erfüllen die Rechtsnormen diese Anforderungen, wird willkürlicher Zwangsausübung der jeweiligen Regierung vorgebeugt und dem einzelnen ein großer Spielraum erlaubter Handlungen gesichert ${ }^{29}$. Er kann seine eigenen Ziele verfolgen und dabei seine individuellen Fertigkeiten und Kenntnisse nutzen. Die Regeln des Rechts schaffen für ihn Rechtssicherheit; der einzelne kennt seine Rechte und Pflichten sowie die seiner Mitbürger, ebenso die Konsequenzen regelwidrigen Verhaltens. Zusammen mit seinen persönlichen Fertigkeiten und Kenntnissen kann er die Rechtsnormen als Grundlage seiner Entscheidungen verwenden. Sie sagen ihm, mit welchem Verhalten anderer er rechnen kann und erweitern dadurch den Bereich, in dem er die Folgen seines eigenen Handelns voraussehen kann. Die Regeln des Rechts bilden eine verlässliche Grundlage, die es dem einzelnen ermöglicht, seinen geschützten Bereich zu bestimmen und das Verhalten seiner Mitbürger abzuschätzen. Sie erleichtern es den Menschen damit zu planen, zu kooperieren und ihre Handlungen aufeinander abzustimmen ${ }^{30}$. Auf diese Weise ermöglicht das Recht die spontane Bildung und Aufrechterhaltung einer komplexen Ordnung menschlichen Handelns, die Millionen von Menschen umfassen kann. Jeder einzelne hat in einer solchen Ordnung die Möglichkeit, seine persönlichen Ziele zu verfolgen, und gute Chancen, sie zu erreichen. Gleichzeitig gewährleisten Rechtsnormen der genannten Art, dass die Menschen bei der Verfolgung ihrer Interessen zur Bedürfnisbefriedigung anderer Menschen beitragen. Durch die Rechtsnormen wird das eigeninteressierte Handeln der Menschen so kanalisiert, dass es der Gesellschaft nicht schadet, sondern nutzt. Der einzelne kann seine Ziele nämlich nur erreichen, indem er den geschützten Bereich der anderen Individuen respektiert und ihnen Güter zum Tausch anbietet, die diese tatsächlich nachfragen. In der durch die Herrschaft des Rechts geschaffenen Handelnsordnung nutzen die Bürger ihr persönliches Wissen zum Wohle der gesamten Gesellschaft, Ressourcen werden effizient alloziiert, die Pläne und Handlungen der Menschen effizient koordiniert. Dadurch wird ein großer Wohlstand erwirtschaftet, der die Ernährung einer großen, zunehmenden Zahl von Menschen ermöglicht.

29 Vgl. John Locke, Two Treatises of Government: In the Former, The False Principles and Foundation of Sir Robert Filmer, And His Followers, are Detected and Overthrown. The Latter is an Essay concerning The True Original, Extent, and End of Civil-Government (1690), 2. Aufl., Cambridge 1988, S. $373 \mathrm{ff}$.

30 Vgl. David Hume, "A Treatise of Human Nature: Being an Attempt to Introduce the Experimental Method of Reasoning into Moral Subjects« Vol. III: »Of Morals« aaO. (FN 3), S. 293 ff. 
Die Herrschaft des Rechts erhöht nicht nur den Wohlstand der betreffenden Gesellschaft, sondern hilft ihr auch, im Wettbewerbsprozess der kulturellen Evolution zu bestehen. Änderungen der Umfeldbedingungen betreffen oft nur Teile der Gesellschaft und werden anfangs nur wenigen Individuen bekannt. Für die Prosperität der Gesellschaft und unter Umständen sogar für ihr Überleben ist es aber von entscheidender Bedeutung, dass sich diese Individuen so rasch wie möglich unter Nutzung ihres speziellen Wissens an diese Änderungen anpassen. Weil die Regeln des Rechts allgemein, abstrakt, bekannt und gewiß sind, können sich die Individuen mit Hilfe dieser Regeln und unter Nutzung ihrer Kenntnisse der »besonderen Umstände von Ort und Zeit « ${ }^{31}$ flexibel an die Veränderungen ihres jeweiligen Umfeldes anpassen; zugleich wird ihr Handeln durch die Regeln so kanalisiert, dass sie dies zum Nutzen der gesamten Gesellschaft tun. Auf diese Weise werden exogene Störungen rasch dezentral absorbiert.

Anpassungen an veränderte Umfeldbedingungen können nicht nur in Veränderungen individuellen Handelns und der gesellschaftlichen Handelnsordnung bestehen, sondern auch in der Erfindung neuer oder der Modifikation bestehender »innerer " Institutionen, etwa in der Entwicklung neuer oder der Modifikation existierender Formen der Unternehmensorganisation ${ }^{32}$. Auch dadurch können exogene Änderungen absorbiert sowie der Wohlstand und das Überleben der Gesellschaft gesichert werden. Allgemeine abstrakte Rechtsnormen erleichtern die Evolution solcher »innerer" Institutionen und erhöhen auf diese Weise die Anpassungsfähigkeit der Gesellschaft noch weiter.

Da sich die meisten Regeln des Rechts evolutionär entwickelt haben (selbst solche, die ursprünglich bewusst eingeführt wurden), verkörpern sie Ergebnisse früherer Erfahrungen, die genutzt werden, solange die Menschen nach diesen Regeln handeln. Dabei handelt es sich oft um heute nicht mehr bewusste Erfahrungen vieler Generationen. Die Nutzung des in solchen Rechtsnormen gespeicherten Wissens ist möglich, obwohl das Wissen von den Vorzügen, die eine bestimmte allgemeine Regel unter verschiedenen Umständen hat, sehr beschränkt ist, vor allem das artikulierbare Wissen. Die Regeln stellen evolutionäre Anpassungen der ganzen Gesellschaft an ihr Umfeld und an die Wesenszüge ihrer Mitglieder dar. Im Laufe der kulturellen Evolution evolutionär gewachsene allgemeine Rechtsnormen verkörpern Wissen über die zweckmäßigste Regelung des Zusammenlebens, die sich im Laufe der Zeit unter wechselnden Umständen bewährt hat. Sie sind wichtige Manifestationen der kulturellen Evolution und erleichtern diese, sofern sie die genannten Bedingungen erfüllen. Auch deswegen sind sie für eine Gesellschaft notwendig und hilfreich, um im Prozess der kulturellen Evolution zu bestehen.

$\mathrm{Da}$ das Wissen, das in einer Gesellschaft zur Aufrechterhaltung der komplexen, anpassungsfähigen und wohlfahrtsschaffenden Handelnsordnung teils in überliefer-

31 Friedrich A. von Hayek, »Die Verwertung des Wissens in der Gesellschaft« aaO. (FN 26), S. 107.

32 Vgl. Ludwig M. Lachmann, »Wirtschaftsordnung und wirtschaftliche Institutionen « in: ORDO, Bd. 14 (1963), S. 63-77. 
ten Rechtsnormen gespeichert, teils auf die Gesellschaftsmitglieder verstreut ist, und da das in überlieferten Rechtsnormen gespeicherte Wissen nur teilweise bekannt ist und da sich das auf die Gesellschaftsmitglieder verstreute Wissen nicht zentralisieren lässt, können Anpassungen einer solchen Handelnsordnung und »innerer « Institutionen an veränderte Umstände nicht zentral durch den Staat vorgenommen werden. Vielmehr müssen traditionelle Rechtsnormen grundsätzlich respektiert werden, und das Handeln der Menschen darf nur durch allgemeine, bekannte und gleichbehandelnde abstrakte Regeln beschränkt werden. Nur so kann sich eine ausgedehnte Gesellschaft effizient an Umstände anpassen, die sich laufend auf nicht prognostizierbare Weise ändern. Die Alternative bestünde in einer Abfolge diskretionärer Einzelentscheidungen und Einzeleingriffe des Staates in die spontane Handelnsordnung. Dadurch würden jedoch die adaptiven, wohlfahrtssteigernden Wirkungen der Rechtsnormen nicht zur Entfaltung kommen können; Handelnsordnung und Kulturevolution würden erheblich gestört.

Obwohl der Staat daher an die Herrschaft des Rechts gebunden sein sollte, stehen ihm im Rahmen dieses Prinzips als Gesetzgeber und in der Rechtsprechung wichtige Regelungsbereiche offen, so etwa das Vertrags-, das Arbeits-, das Schadens- und das Verwaltungsrecht ${ }^{33}$. Auch bestehen bei der Entwicklung konkreter Rechtsnormen nicht unbeträchtliche Gestaltungsspielräume. Die Anforderungen, die aus Sicht der kulturellen Evolution an die rechtlichen Regeln gestellt werden müssen, sind abstrakter Natur; sie können durch unterschiedlichste Regeln erfüllt werden. Der konkrete Inhalt der Regeln kann und sollte je nach kulturellen Traditionen und Präferenzen der Menschen von Land zu Land variieren. Da daneben ein weiter Spielraum der Individuen bei der Nutzung der Regeln als Instrumente zur Verfolgung ihrer unterschiedlichen individuellen Ziele besteht, ist mit der Herrschaft des Rechts eine große Bandbreite rechtlicher Regeln sowie persönlicher Ziele und Handlungsformen vereinbar.

An den rechtlichen Normen sollte die Politik grundsätzlich konsequent festhalten, weil die Menschen ihr Verhalten darauf einstellen und daher die Erhaltung und Anpassungsfähigkeit der gesamten spontanen Handelnsordnung von der Verlässlichkeit und Konstanz der rechtlichen Rahmenbedingungen abhängt. Zurückhaltung bei der Änderung von Rechtsnormen sollte auch geübt werden, weil diese darüber hinaus mit den historisch gewachsenen, tief verwurzelten und sich nur langsam wandelnden Moralvorstellungen der jeweiligen Gesellschaft in Einklang stehen müssen. Gleichwohl sind Reformen des Rechts notwendig, wenn das herkömmliche Recht Mängel zeigt oder geänderte Umfeldbedingungen Reformen erforderlich machen. Da die spontane Ordnung jeder ausgedehnten Gesellschaft auf einer Tradition überlieferter rechtlicher und moralischer Normen beruht, deren Bedeutung die Menschen nicht vollständig verstehen können, muss man dabei aber auf dieser Tradition aufbauen. Neue und novellierte Rechtsnormen müssen mit den übrigen Regeln konsistent und kompatibel sein; sie müssen einen wirkungsvollen Beitrag zur

33 Ähnlich Heiko Geue, »Sind ordnungspolitische Reformanstrengungen mit Hayeks Evolutionismus vereinbar?« in: ORDO, Bd. 49 (1998), S. 156. 
Erhaltung und Anpassungsfähigkeit der Gesamtordnung der Handlungen leisten, der alle anderen Regeln dienen ${ }^{34}$.

\subsection{Subsidiarität}

Die dritte Leitlinie besagt, dass die Staatstätigkeit eng begrenzt und dezentral organisiert sein sollte. Folgende Funktionen des Staates sind dabei allerdings unerlässlich:

- Der Staat muss die Freiheit seiner Bürger anerkennen und schützen - etwa die Meinungs-, Vertrags- und Gewerbefreiheit.

- Der Staat muss das Eigentum seiner Bürger anerkennen und schützen. Er muss Regeln festlegen, nach denen die Individuen Eigentum erwerben und übertragen können. Und er muss gewährleisten, dass die Bürger ihre Eigentumsrechte wahrnehmen können.

- Der Staat muss freiwillig geschlossene Verträge durchsetzen - vorausgesetzt natürlich, sie verstoßen nicht gegen Recht oder Moral.

Diese und einige weitere Aufgaben hat der Staat in erster Linie mit Hilfe des Rechts zu erfüllen. Die rechtlichen Regeln sollten so gestaltet sein, dass sie den Gesellschaftsmitgliedern die bestmöglichen Bedingungen bieten, ihre eigenen Ziele zu erreichen. Zu diesem Zweck müssen sie auch den in Abschnitt 3.2. genannten Anforderungen genügen. Vor allem staatliche Zwangsmaßnahmen müssen mit der Herrschaft des Rechts vereinbar sein. Solche Maßnahmen sind auf die Durchsetzung allgemeiner, abstrakter, bekannter und gleichbehandelnder Rechtsnormen zu beschränken. Prinzipiell unstatthaft dagegen müssen Maßnahmen sein, mit denen der Staat unter Verwendung seiner Zwangsgewalt gezielt bestimmte Individuen oder Gruppen benachteiligt oder begünstigt, etwa durch Handelsbeschränkungen oder Privilegien. Unstatthaft müssen auch Rechtsnormen sein, die zwar allgemein, abstrakt, bekannt und gleichbehandelnd sind, aber trotzdem die Freiheits- oder Eigentumsrechte der Bürger stark beschneiden - etwa Steuergesetze, die die Eigentumsrechte der Bürger durch eine hohe Steuerbelastung aushöhlen.

Neben der Durchsetzung allgemeiner freiheitssichernder Rechtsnormen sollte der Staat noch für die Bereitstellung bestimmter essentieller Güter Sorge tragen, die von Privatunternehmen nicht auf eigene Initiative angeboten werden - zumeist, weil es sich dabei um öffentliche Güter handelt, bei denen es entweder unmöglich oder zu schwierig ist, den einzelnen Nutznießer dafür zahlen zu lassen. Die staatliche Bereitstellung solcher Güter sollte jedoch - abgesehen von der zu ihrer Finanzierung erforderlichen Steuererhebung - keinen Zwangscharakter besitzen. Vor allem sollte dem Staat kein Monopol eingeräumt werden; Privatunternehmen sollten stets die Möglichkeit besitzen, diese Güter ebenfalls anzubieten. Generell sollte der

34 Vgl. Friedrich A. von Hayek, Recht, Gesetzgebung und Freibeit. Eine neue Darstellung der liberalen Prinzipien der Gerechtigkeit und der politischen Ökonomie, Bd. II: Die Illusion der sozialen Gerechtigkeit, aaO. (FN 3), S. 41 ff. 
Staat bei der Bereitstellung dieser Güter, wie auch sonst, den selben Rechtsnormen unterworfen sein wie die privaten Wirtschaftssubjekte. Darüber hinaus sollten die staatlichen Güter der Ausübung der individuellen Freiheitsrechte der Bürger förderlich sein; sie sollten einen günstigen Rahmen für individuelle Dispositionen schaffen. Bevor der Staat ein Gut bereitstellt, sollte zudem sichergestellt sein, dass dessen Nutzen die mit ihm verbundenen Kosten deutlich überwiegt. Dabei ist zu berücksichtigen, dass Behörden und Staatsunternehmen, die üblicherweise keinem Konkursrisiko ausgesetzt sind, zu einer ineffizienten Wirtschaftsweise tendieren. Daher sollte der Staat die entsprechenden Güter möglichst nicht selbst produzieren, sondern mit Hilfe wettbewerblicher Verfahren (wie etwa Ausschreibungen oder Auktionen) Privatunternehmen auswählen, die dies im staatlichen Auftrag möglichst kostengünstig übernehmen. Und schließlich sollten sich die staatlichen Aktivitäten auf eine geringe Zahl von Gütern beschränken, um den staatlichen Machtbereich nicht über das Notwendigste auszudehnen. Beispiele für Güter, die der Staat bereitstellen sollte, sind Infrastruktur, Bildung, soziale Sicherung und stabiles Geld.

Um die Staatstätigkeit wirksam zu begrenzen, sollten entsprechende Vorschriften in die Verfassung aufgenommen werden. Solche konstitutionellen Normen können (a) die Art der Aufgaben, die der Staat erfüllen soll, bzw. die Art der Güter, die er bereitstellen soll, beschränken und/oder (b) den Umfang staatlicher Einnahmen oder Ausgaben quantitativ begrenzen (etwa in Form von Obergrenzen in Prozent des Bruttoinlandsprodukts) $)^{35}$.

Der Staat sollte also eine subsidiäre Rolle spielen: Nur dort, wo die Möglichkeiten des einzelnen bzw. einer kleinen Gruppe (z.B. der Familie) nicht ausreichen, eine essentielle Aufgabe zu lösen, sollten staatliche Institutionen eingreifen. Dabei ist Hilfe zur Selbsthilfe Vorrang vor einer unmittelbaren Aufgabenübernahme durch den Staat zu geben. Das Subsidiaritätsprinzip ist auch auf die Aufgabenverteilung innerhalb des Staatssektors anzuwenden: Muss eine Aufgabe vom Staat übernommen werden, ist die Verantwortung für die Aufgabenerfüllung der kleinsten dafür geeigneten Einheit zu übertragen (etwa der Gemeinde).

Durch die enge Begrenzung und dezentrale Organisation der Staatstätigkeit sollte nicht zuletzt auch ein Wettbewerb zwischen verschiedenen Institutionen ermöglicht werden. Durch den großen Freiraum für private Akteure sollten diese beispielsweise die Möglichkeit haben, unterschiedlichste Formen der Unternehmensorganisation oder verschiedene Vertragsformen zu entwickeln und wettbewerblich

35 Notwendigkeit und Möglichkeiten einer konstitutionellen Begrenzung der Staatstätigkeit werden in jüngerer Zeit vor allem im Rahmen der Verfassungsökonomik analysiert. Ein Überblick und eine kritische Würdigung dieses Ansatzes findet sich bei Horst Feldmann, Ordnungstheoretische Aspekte der Institutionenökonomik, aaO. (FN 11), S. 80 ff., 230 ff. Der Verfasser des vorliegenden Aufsatzes hat darüber hinaus sowohl einen konkreten Vorschlag zur konstitutionellen Begrenzung der Steuerbelastung als auch zur Begrenzung der staatlichen Schuldenaufnahme unterbreitet. Vgl. Horst Feldmann, »Konstitutionelle Begrenzung der Steuerbelastung « in: Steuer und Wirtschaft, 75. Jg. (1998), Nr. 2, S. 114-123; ders., »Warum der Stabilitätspakt reformiert werden muß « in: Jabrbuch für Wirtschaftswissenschaften, Bd. 51 (2000), Nr. 3, S. 197-221. 
auf ihre Eignung zu testen. Durch die dezentrale Organisation des Staates sollten die Staatsorgane (z.B. die Gemeinden) in einem Wettbewerb untereinander stehen.

Aus welchen Gründen ist es erforderlich, die Staatstätigkeit auf die genannten Funktionen zu konzentrieren und darüber hinaus eng zu begrenzen? Durch die Sicherung der Freiheit und des Eigentums der Bürger und die Durchsetzung freiwilliger Verträge mit Hilfe allgemeiner Rechtsnormen wird nicht nur das verstreute Wissen der Individuen so genutzt, dass sich die gesellschaftliche Handelnsordnung flexibel und kostengünstig an die sich laufend ändernden äußeren Umstände anpasst; darüber hinaus werden auch Konflikte zwischen Individuen von vornherein vermieden oder zu geringen gesellschaftlichen Kosten beigelegt. Zudem haben die Individuen die Möglichkeit und den Anreiz, ständig neue Güter, Produktionsverfahren, Institutionen usw. zu entwickeln. Wegen der engen Begrenzung der Staatstätigkeit kann die Regierung bzw. die herrschende Mehrheit solche neuen Ideen und Gebräuche nicht unterdrücken. Dies ist deshalb wichtig, weil neue Güter, Produktionsverfahren, Institutionen etc. stets dadurch eingeführt werden, dass zunächst nur eine kleine Minderheit von den Gebräuchen der Mehrheit abzuweichen beginnt und zwar oftmals gegen deren Widerstand. Erst wenn sich die Neuerungen dem Alten als überlegen erweisen, wird auch die Mehrheit zu deren Übernahme bewegt ${ }^{36}$. Durch die Begrenzung der Staatstätigkeit kommt es somit zu mehr Neuerungen, mit denen die Gesellschaft ihren Wohlstand erhöhen und sich besser an geänderte äußere Umstände anpassen kann. Verfügt der Staat dagegen über eine große Machtfülle, werden die Bürger durch zahlreiche Vorschriften gegängelt und wird ein Großteil ihres Einkommens durch hohe Steuern konfisziert, haben sie kaum die Möglichkeit und den Anreiz, sich an geänderte Umstände unter Nutzung ihres jeweiligen Wissens anzupassen, etwa indem sie neue Institutionen entwickeln, die diesen geänderten Umständen besser Rechnung tragen. Damit verliert die Gesellschaft insgesamt an Funktions- und Anpassungsfähigkeit; sie wird anfällig für exogene Schocks oder eine allmähliche Erosion ihrer Wettbewerbsposition im Prozess der kulturellen Evolution. Dabei verringert sich auch ihr Wohlstand oder zumindest dessen Zuwachsrate.

Schädlich sind darüber hinaus Maßnahmen, mit denen der Staat unter Verwendung seiner Zwangsgewalt gezielt bestimmte Individuen oder Gruppen benachteiligt oder begünstigt. Solche Interventionen widersprechen nicht nur der Herrschaft des Rechts; vor allem be- oder verhindern sie die wechselseitige Anpassung der Individuen, auf der die spontane Handelnsordnung und die Evolution der gesellschaftlichen Institutionen beruhen: Diejenigen, die staatlichen Zwangsmaßnahmen ausgesetzt sind, werden daran gehindert, ihre eigenen Ziele zu verfolgen und dabei die ihnen bekannten Umstände zu nutzen. Diejenigen, die in den Genuss staatlicher Vergünstigungen gelangen, werden einseitig von der Notwendigkeit befreit, sich den Umständen anzupassen, denen sie ohne Intervention ausgesetzt wären. Durch solche Interventionen kann daher die Anpassungsfähigkeit, die Evolution und damit eventuell sogar der Bestand der jeweiligen Gesellschaftsordnung gefährdet werden.

36 Vgl. Ludwig Mises, Liberalismus, Jena 1927, S. 48. 
Obwohl der Umfang der Staatstätigkeit aus den genannten Gründen grundsätzlich eng begrenzt sein sollte, ist es wichtig, dass der Staat gleichwohl für die Bereitstellung bestimmter essentieller, insbesondere öffentlicher Güter Sorge trägt, die von Privatunternehmen auf eigene Initiative nicht angeboten werden. Solche Güter können für die internationale Wettbewerbsfähigkeit einer Gesellschaft von entscheidender Bedeutung sein. $\mathrm{Zu}$ denken ist hierbei etwa an eine Infrastruktur und ein Bildungssystem, die der Produktivität der Wirtschaft förderlich sind ${ }^{37}$. Aber auch ein fundamentales Gut wie stabiles Geld ist von eminenter Bedeutung; es sichert nominal fixierte Eigentumsrechte und stellt sicher, dass das System flexibler Marktpreise seine Signal- und Lenkungsfunktion erfüllen kann. Dies ist nicht nur der Effizienz und dem Wohlstand der jeweiligen Gesellschaft zuträglich, vor allem erhöht es ihre Anpassungsfähigkeit, weil die durch Preisänderungen übermittelten Informationen die Wirtschaftssubjekte zu einer den neuen Umständen angemessenen Reaktion veranlassen ${ }^{38}$.

Durch eine konsequente Verwirklichung des Subsidiaritätsprinzips und einen intensiven institutionellen Wettbewerb wird laufend dezentral eine Vielzahl von Problemlösungen generiert, getestet und miteinander verglichen. Ungeeignete Maßnahmen und Institutionen können frühzeitig ausgesondert werden; sie verursachen dadurch nur geringe gesellschaftliche Kosten. Diejenigen Problemlösungen, die sich als überlegen erweisen, können wiederum allgemein übernommen werden; sie breiten sich in der Gesellschaft aus. Dieser Prozess des Experimentierens, der permanenten Hervorbringung einer Vielzahl insbesondere institutioneller Alternativen, ihrer Selektion im Wettbewerb und der Verbreitung der am besten geeigneten ist für die Anpassungsfähigkeit einer Gesellschaft im Prozess der kulturellen Evolution vor allem deshalb bedeutsam, weil es bei der bewussten Gestaltung, aber auch bei der ungeplanten Evolution von Institutionen immer wieder zu Fehlentwicklungen kommen kann. So können sich planmäßig entworfene Institutionen in der Praxis als untauglich erweisen. Auch kann sich die institutionelle Evolution in einzelnen Bereichen ungeplant in eine Richtung entwickeln, die sich erst nach einer gewissen Zeit als falsch herausstellt. Zudem können Evolutionshemmnisse auftreten. Je intensiver jedoch der institutionelle Wettbewerb, desto mehr institutionelle Alternativen werden generiert, desto stärker ist der Selektionsdruck und desto größer ist damit die Wahrscheinlichkeit, dass ineffiziente Institutionen frühzeitig entdeckt und ausgesondert sowie Evolutionssackgassen verlassen bzw. Evolutionshemmnisse überwunden werden. Voraussetzung für die Funktionsfähigkeit des institutionellen

37 Vgl. Horst Siebert, Zum Paradigma des Standortwettbewerbs, Tübingen 2000.

38 Der Autor des vorliegenden Aufsatzes hat ein Entlohnungssystem für die Mitglieder des Rates der Europäischen Zentralbank entwickelt, das diese wirksam und nachhaltig zu einer strikt stabilitätsorientierten Geldpolitik anhalten könnte. Ein solches System ist vor allem deswegen notwendig, weil in vielen Mitgliedsländern der Europäischen Währungsunion noch nicht die gleiche Stabilitätskultur existiert wie in Deutschland. Vgl. Horst Feldmann, "Stabilitätsanreize für Europas Zentralbanker « in: Wirtschaftsdienst, 78. Jg. (1998), Nr. 2, S. 121-128. 
Wettbewerbs ist freilich eine Gesellschaft, die nach außen und innen stets offen bleibt für Neuerungen.

Die Umsetzung des Subsidiaritätsprinzips kann nicht auf beliebige Weise erfolgen. Sie hat sich in erster Linie an den Erfordernissen der kulturellen Evolution auszurichten. Daher muss es möglichst konsequent umgesetzt werden. Beispielsweise muss der Umfang der Staatstätigkeit durch geeignete Verfassungsvorschriften wirksam beschränkt werden; der institutionelle Wettbewerb muss nicht nur zugelassen, seine Ergebnisse dürfen auch nicht durch andere Maßnahmen der Politik konterkariert werden. Soweit freilich den Anforderungen der kulturellen Evolution Genüge getan ist, besteht ein nicht unerheblicher Gestaltungsspielraum. Er kann in einer Weise genutzt werden, die den Präferenzen der Gesellschaftsmitglieder entspricht. Welche öffentlichen Güter beispielsweise in welcher Qualität und Quantität bereitgestellt, von wem sie produziert und wie sie finanziert werden, kann, soweit den Erfordernissen der kulturellen Evolution Rechnung getragen wird, nach den Präferenzen der Gesellschaftsmitglieder entschieden werden.

\section{Fazit}

Obwohl der demokratische Wettbewerb alternativer politischer Konzepte, wie ausgeführt, ein wichtiges Mittel darstellt, mit dessen Hilfe eine Gesellschaft im Prozess der kulturellen Evolution bestehen kann, unterscheiden sich die politischen Implikationen der kulturellen Evolution in einer Hinsicht fundamental von der Rolle, die die Politik nach heute herrschender Meinung in der Demokratie spielen sollte. Es gilt heute als selbstverständlich, dass die Politik ausschließlich den jeweiligen Willen des Volkes umzusetzen hat; die Präferenzen der Bürger sollen in der Politik möglichst gut zum Ausdruck kommen ${ }^{39}$. Aus Sicht der kulturellen Evolution muss das primäre, übergeordnete Ziel der Politik jedoch die Überlebensfähigkeit der jeweiligen Gesellschaft sein, für die die Politik Verantwortung trägt, nicht der jeweilige Wille des Volkes ${ }^{40}$. Zwar entsprechen die Wünsche der Menschen vielfach dem, was die kulturelle Evolution erfordert; dies ist aber durchaus nicht immer der Fall. So werden zur Verwirklichung der Idee der »sozialen Gerechtigkeit« und zur Befriedigung der Wünsche einflussreicher Interessengruppen heute vielfach politische Maßnahmen gefordert und durchgeführt, die mit den Anforderungen, die die kulturelle Evolution mit sich bringt, unvereinbar sind. Wenn eine Gesellschaft jedoch diesen Anforderungen nicht entspricht, läuft sie Gefahr, ihren Wohlstand einzubüßen und im Extremfall sogar unterzugehen. Angesichts der entscheidenden Rolle, die die ge-

39 Siehe etwa Arno Waschkuhn, Demokratietheorien. Politiktheoretische und ideengeschichtliche Grundzüge, München 1997.

40 Dies bedeutet selbstverständlich nicht, dass hiermit irgendeiner Form autoritärer oder totalitärer politischer Herrschaft das Wort geredet wird. Eine solche Form politischer Herrschaft stünde im Gegensatz zu den im vorliegenden Aufsatz entwickelten Leitlinien. Statt dessen empfiehlt sich eine geeignete verfassungsmäßige Verankerung dieser Leitlinien. 
nannten Institutionen beim Aufstieg der westlichen Welt gespielt haben, und des starken Selektionsdrucks, den die kulturelle Evolution heute ausübt, sollten die hier entwickelten Leitlinien konsequent verwirklicht werden - selbst wenn dies manchen unmittelbaren Wünschen der Menschen widerspricht. Nur insoweit den Anforderungen der kulturellen Evolution Genüge getan wird, besteht Spielraum, die Wünsche der Bürger zu befriedigen.

\section{Zusammenfassung}

Im Laufe der kulturellen Evolution haben sich in der westlichen Welt Institutionen entwickelt und durchgesetzt, die zu einer früher unbekannten Zunahme des Wohlstands und der Bevölkerung geführt haben. Im vorliegenden Aufsatz werden zunächst die Charakteristika und die Bedeutung der kulturellen Evolution herausgearbeitet. Anschließend werden der Spielraum und die Rolle der Politik in der kulturellen Evolution grundsätzlich beleuchtet sowie einige in diesem Zusammenhang weit verbreitete Irrtümer ausgeräumt. Im letzten Schritt schließlich werden aus den Charakteristika der kulturellen Evolution drei konkrete Leitlinien abgeleitet, die die Politik beachten sollte: Erstens sollte das Prinzip der individuellen Freiheit umfassend verwirklicht sein. Zweitens sollte die Herrschaft des Rechts gewährleistet sein. Drittens sollte die Staatstätigkeit eng begrenzt und dezentral organisiert sein. Wie im vorliegenden Aufsatz gezeigt wird, sind die Chancen einer Gesellschaft, im Wettbewerbsprozess der kulturellen Evolution zu bestehen und zu prosperieren, um so größer, je konsequenter diese Leitlinien befolgt werden.

\section{Summary}

In the course of cultural evolution, certain institutions have emerged and become dominant in the Western world that have led to an unprecedented rise in prosperity and population. This paper first explains the characteristics and significance of cultural evolution. Subsequently, it explains the fundamental role of politics and the scope for political action in cultural evolution, clearing up some fairly wide-spread misconceptions about this question. Finally, it derives three specific guidelines from the characteristics of cultural evolution that should be followed in politics. First, the principle of individual freedom should be realized as far as possible. Second, the rule of law should be secured. Third, the scope of action of the state should be closely limited and the state should be decentrally organized. The more consistently these guidelines are followed, the better the prospects of the respective society to survive and prosper in the competitive process of cultural evolution. 